\title{
APPLICATION OF LYOCELL FIBER STRUCTURE FORMATION MECHANISM IN FLAME-RETARDANT MODIFICATION OF LYOCELL FIBER
}

\author{
GESHENG YANG, ${ }^{*}$ YONGWEI MENG, ${ }^{* *}$ HUIHUI ZHANG* ${ }^{*}$ and HUILI SHAO* \\ "State Key Laboratory for Modification of Chemical Fibers and Polymer Materials, \\ College of Material Science and Engineering, Donghua University, Shanghai 201600, China \\ ** Shandong Lulian New Material Co., Ltd., Shandong 255000, China \\ \Corresponding author: Gesheng Yang, gsyang@dhu.edu.cn
}

Received May 11, 2020

In order to overcome the disadvantage of Lyocell fiber flammability, two types of flame-retardant finishing liquids, 2carboxyethyl phenylphosphic acid (CEPPA) and N-hydroxymethyl-3-dimethoxyphosphoacyl propanamide (MDPA), were used in this study to treat Lyocell fiber in two different states: never-dried and dry. The results showed that CEPPA and MDPA can react with the hydroxyl groups of the cellulose and graft onto the Lyocell fiber under appropriate conditions, resulting in increased flame-retardant performance of the fiber, a slight reduction in crystallinity, and a significant decline in mechanical properties. Compared with the dry fiber, the P content and LOI of the fiber obtained by treating the as-spun never-dried Lyocell fiber rose significantly: the P content was higher by $38.9 \%$ (for CEPPA) and $20.5 \%$ (for MDPA), respectively, while the LOI increased by $6.0 \%$ (for CPPA) and $4.0 \%$ (for MDPA), respectively, which means that the fiber had better flame-retardant performance. Although the breaking strength of the fiber decreased, it still met the requirements for textiles. In addition, direct flame-retardant treatment of never-dried wet fiber can reduce energy consumption by avoiding repeated drying. Furthermore, the results of this study also have guiding significance for other post-processing procedures for Lyocell fibers, such as dyeing, catalyst infiltration during carbon fiber preparation etc.

Keywords: Lyocell fiber, flame-retardant fiber, treatment, crystallinity

\section{INTRODUCTION}

Lyocell fiber is regenerated from cellulose by using the dry-jet wet spinning process, where the $\mathrm{N}$-methylmorpholine-N-oxide monohydrate solution is used as dissolving agent. ${ }^{1}$ In contrast to the conventional viscose process, the Lyocell process is a relatively simple, resource preserving, and environmentally friendly way to produce regenerated cellulose fibers without derivatization and with a reduced number of processing steps. In addition, compared with other regenerated cellulose fibers, Lyocell fiber has better mechanical properties, mainly due to its tighter aggregate structure and higher crystallinity. The structure formation and structure-property relation of the Lyocell fibers, involving the deformation of the cellulose-NMMO spinning dope in the spinneret and the air gap, the coagulation of the cellulose, and the washing and drying of the fiber, have been investigated by many researchers. ${ }^{2-6}$ During the shaping of the highly viscous dope, the structure formation is determined by the concurrent orientation, coagulation and crystallization processes. The crystallization is influenced by the solution characteristics, the precipitation conditions, as well as the drying and post-treatment conditions. These process parameters are not independent from each other. ${ }^{7}$

The formation of the crystalline structure of Lyocell fiber was studied by Weigel et al. ${ }^{2}$ Based on on-line measurements of the crystalline structure of never-dried Lyocell fiber in the process of spinning using a synchrotron, they found that the characteristic diffraction peak at $2 \theta \approx 11.8^{\circ}$, corresponding to the 101 crystal plane of cellulose II, was very low. Therefore, they

Cellulose Chem. Technol., 54 (9-10), 1015-1022(2020) 
considered that the ordered arrangement supermolecular structure of cellulose had not been fully formed before Lyocell fiber was dried, especially the lateral ordered structure of the 101 crystal plane had not been completely formed. However, their work could not clearly reflect the evolution of the crystalline structure of Lyocell fiber. Wei et al. used the wide-angle X-ray diffraction technique to study the formation process of the crystalline structure of as-spun Lyocell fiber that had never been dried, and compared it with the crystalline structure of dried and re-wetted Lyocell fiber. ${ }^{8}$ The experimental results showed that the three-dimensional ordered supramolecular structure in the never-dried asspun Lyocell fibers had not been completely formed, the crystallinity of the fibers was low, and the quasicrystal content was relatively high. With the decrease of the water content in the fiber, some of the quasicrystals in the fiber gradually transformed into the main crystal structure of cellulose II, and the crystallinity and the grain size of each major crystal plane increased in an ' $\mathrm{S}$ ' shape. That is, during the fiber drying process, the quasicrystal content decreased rapidly and the crystallinity increased rapidly with the decrease of the water content. When the water content was less than $35 \%$, a relatively perfect crystalline structure had basically formed, and the crystallinity was basically the same as that of the dry Lyocell fiber. Researchers at the Thuringia Institute for Plastics and Textiles (TITK) swelled dry Lyocell filaments using different media, and conducted further tensile studies. ${ }^{9}$ As a result, they found that after the water-swellable filaments were stretched, the modulus of elasticity significantly increased by up to $50 \%$. When the filaments swollen by $\mathrm{NaOH}$ were stretched, the modulus of elasticity could increase by up to $80 \%$. On the other hand, in order to improve the mechanical properties of Lyocell fibers, Zhang et al. rewetted dry Lyocell fibers and then heattreated them, in order to examine the durability of their properties. They found that the degree of orientation of the amorphous regions of the fibers increased after the heat treatment, which temporarily improved the mechanical properties of the fibers. However, because no new crystalline structure was formed in the fibers to fix this oriented structure perfected by the heat treatment, the improved mechanical properties could not be sustained. ${ }^{10}$ After rewetting, water molecules only enter the amorphous region of the fiber, which has little effect on the crystalline structure of Lyocell fibers. If the structure of Lyocell fibers is to be changed by post-treatment, the post-treatment must be performed when the fiber has never been dried and the moisture content is greater than $35 \%$.

Lyocell fiber is a new type of cellulose fiber with many excellent properties, but its limiting oxygen index (LOI) is only about $18 \%$, which makes it a flammable fiber, and limits its application in some fields. In order to improve the flame retardancy of Lyocell fibers, Delhom et al. added organically modified montmorillonite nanoparticles to a cotton cellulose/NMMO solution to prepare flame-retardant Lyocell fibers through blend spinning. However, if the amount of the inorganic flame retardant is too large, it will affect the spinnability of the solution and the washing resistance of the fiber. ${ }^{11}$ Seddan et al. used Pyrovatex CP as a flame retardant and melamine as a cross-linking agent to perform a flame-retardant treatment on Lyocell fibers. The LOI of the treated fiber reached $40 \%$, but melamine decomposition would release a higher content of formaldehyde. ${ }^{12}$ Mengal et al. used citric acid instead of melamine as a cross-linking agent to prepare flame-retardant Lyocell fibers. Although the formaldehyde emission was reduced by $25 \%$, the flame-retardant performance was also reduced. ${ }^{13}$ Liu et al. used ammonium phytate synthesized by the reaction between phytic acid and urea as a flame retardant to treat Lyocell fibers, and obtained Lyocell fibers with better flame retardancy, but the mechanical properties of the fibers after the treatment were poor. ${ }^{14}$

Generally, during the post-treatment process, the flame retardant can only penetrate into the amorphous region of the fiber and can react with the cellulose molecules in the amorphous region under appropriate conditions. Therefore, higher crystallinity will seriously affect the graft amount of the flame retardant. On the contrary, the lower crystalline structure promotes the contact of the flame retardant with the cellulose molecules, thereby improving the subsequent grafting reaction. Based on the formation mechanism and the characteristics of the Lyocell fiber structure, two kinds of flame-retardant finishing liquids, 2carboxyethyl phenylphosphic acid (CEPPA) and N-hydroxymethyl-3-dimethoxyphosphoacyl propanamide (MDPA), were used in this study to directly treat never-dried as-spun Lyocell fiber obtained from spinning. The aim was to further improve the flame retardancy of Lyocell fibers 
and reduce energy consumption caused by multiple drying processes.

\section{EXPERIMENTAL}

Materials

Wood pulp, with a degree of polymerization of 547 and $\alpha$-cellulose of $88 \%$, was provided by Weyerhaeuser (USA); N-methylmorpholine-N-oxide (NMMO) aqueous solution (50 wt $\%$ ) was obtained from BASF (Germany). Antioxidant, N-propyl gallate, was purchased from Shanghai Chemical Corporation (China).

Flame retardants, 2-carboxyethyl phenylphosphic acid (CEPPA) and N-hydroxymethyl-3dimethoxyphosphoacyl propanamide (MDPA), were provided by Zibo Zhuxin Chemical Co. Ltd. (China) and by Jiangsu Liside Chemical Co. Ltd. (China), respectively. Hexamethylolmelamine (HMM) as crosslinking agent, of chemical purity, was supplied by Chongqing Jianfeng Haokang Chemical Co. Ltd. (China). Phosphoric acid, of analytical grade, was provided by Shanghai Lingfeng Chemical Reagent Co. Ltd. (China).

\section{Preparation of Lyocell fiber}

A NMMO aqueous solution of $50 \mathrm{wt} \%$ was concentrated to $74 \mathrm{wt} \%$ by vacuum distillation. The weighed distilled solution and wood pulp were mixed and stirred in a dissolving tank at $95^{\circ} \mathrm{C}$. At the same time, vacuum distillation was performed again until the molar ratio of NMMO to water reached $1: 1$, then a brown-yellow cellulose solution with 12 wt $\%$ of cellulose was prepared. The obtained cellulose solution was extruded through a spinneret with 100 orifices (each orifice was $80 \mathrm{~lm}$ in diameter) at $90{ }^{\circ} \mathrm{C}$ by a metering pump, and then passed through an air gap, a coagulation bath (water), and a water bath (to completely remove NMMO), and finally, Lyocell fiber was obtained at a spinning speed of $90 \mathrm{~m} / \mathrm{min}^{15}$ During the spinning process, 4 strands of fiber were obtained, ensuring consistent weight by controlling the winding time. Among them, for 2 strands of fibers, there was continuous water dripping during the winding process to keep them wet, and the other 2 strands of fibers were air-dried.

\section{Flame-retardant finishing of Lyocell fiber}

CEPPA and MDPA were formulated into a flameretardant finishing solution, with a concentration of $8 \%$ and $40 \%$, respectively, and an appropriate amount of auxiliary components was added. At a certain temperature, the above-mentioned 4 strands of dry and wet Lyocell fibers were, respectively, immersed into the flame-retardant finishing solution, then passed through a laboratory padder with two dips and two nips, then the fibers were microwave-treated for 3 minutes, pre-dried at $90{ }^{\circ} \mathrm{C}$ for 3 minutes, cured in a Mathis curing oven at $160{ }^{\circ} \mathrm{C}$ for $5 \mathrm{~min}$, and finally soaped at $60{ }^{\circ} \mathrm{C}$ (soap powder concentration: $5 \mathrm{~g} / \mathrm{L}$ ) for 3 minutes, washed and dried to obtain flame-retardant Lyocell fiber. The sample processing conditions and the corresponding codes are shown in Table 1.

\section{Characterization of structure and properties of flame-retardant Lyocell fiber \\ FTIR analysis}

Infrared spectra were taken on a Nicolet 6700 FTIR spectrophotometer (Nicolet Instrument Corp., Madison, WI, USA) in the wavelength range from 4000 to 400 $\mathrm{cm}^{-1}$. Samples were prepared as a thin film with potassium bromide. The obtained spectra were the result of 24 scans at the resolution of $4 \mathrm{~cm}^{-1}$.

\section{Thermogravimetric analysis (TG)}

The thermogravimetric analysis of the fiber was performed on a STA409PC TG\&DSC synchronous thermal analyzer, with sample weight of $5 \sim 10 \mathrm{mg}$, air atmosphere, heating rate of $10{ }^{\circ} \mathrm{C} / \mathrm{min}$ and in the temperature range of $50 \sim 700{ }^{\circ} \mathrm{C}$.

\section{$X$-ray diffraction analysis}

The crystal structure of the fiber samples was analyzed by a D/max-2550PC X-ray diffractometer from Rigaku, Japan. Test conditions: reflection method, CuKa target, $40 \mathrm{Kv}, 200 \mathrm{~mA}, 2$ theta $=5-40^{\circ}$.

\section{Elemental analysis}

The American Leeman Prodigy Inductively Coupled Plasma Atomic Emission Instrument was used to test the P content of flame-retardant fibers.

\section{Analysis of the mechanical properties of fibers}

The fiber fineness was tested by an XD-1 fiber fineness meter (developed by Donghua University). The test range was $0.8 \sim 40$ dtex, the clamping length was $20 \mathrm{~mm}$, the measurement error was $\leq \pm 2 \%$, and the test environmental conditions were: $20{ }^{\circ} \mathrm{C}, 65 \%$ $\mathrm{RH}$.

Table 1

Sample processing conditions and corresponding codes

\begin{tabular}{lcc}
\hline Sample code & Flame-retardant finishing solution & State of Lyocell fiber \\
\hline Untreated fiber & $/$ & Dry \\
CEPPA-wet & $8 \%$ CEPPA & Never-dried \\
CEPPA-dry & $8 \%$ CEPPA & Dry \\
MDPA-wet & $40 \%$ MDPA & Never-dried \\
MDPA-dry & $40 \%$ MDPA & Dry \\
\hline
\end{tabular}


The breaking strength and initial modulus of the fiber were measured by an XD-1 monofilament strength tester (developed by Donghua University). The clamping length of the fiber was $10 \mathrm{~mm}$, and the tensile rate was $5 \mathrm{~mm} / \mathrm{min}$. The test environment conditions were as follows: $20{ }^{\circ} \mathrm{C}, 65 \% \mathrm{RH}$. Each fiber sample was tested 20 times, and the average value was considered.

\section{Flame-retardant performance analysis}

The flame-retardant performance of the Lyocell fiber was tested according to the "oxygen index method of textile combustion performance test" GB/T 5454-1997.

\section{RESULTS AND DISCUSSION \\ Infrared spectrum analysis}

The active hydroxyl group on the Lyocell fiber can react chemically with the carboxyl group on the flame retardant CEPPA, under certain conditions, to form an ester group; the structural formula is shown in Scheme 1. In addition, it can directly react with the N-hydroxymethyl on the flame retardant MDPA, under certain conditions, and be firmly bonded with covalent bonds. The flame retardant MDPA can also be grafted onto the Lyocell fiber through the cross-linking agent $\mathrm{HMM} ;{ }^{16}$ the structural formula is shown in Scheme 2.

The structure of the Lyocell fiber before and after treatment was investigated by FTIR spectroscopy, as shown in Figure 1. Compared with the spectrum of the untreated fiber, the curve CEPPA-dry in Figure 1 shows a small vibration peak near $1730 \mathrm{~cm}^{-1}$, which is attributed to $\mathrm{C}=\mathrm{O}$ stretching vibration from CEPPA, so it can be confirmed that CEPPA has been successfully combined with the Lyocell fibers. In addition, it can be seen from the curve MDPA-dry that a new absorption peak appears near $804 \mathrm{~cm}^{-1}$, this peak is the contribution of triazine, which indicates that the cross-linking agent HMM has combined with the fiber, and the peak at $1551 \mathrm{~cm}^{-1}$ is attributed to -NH- stretching vibration. There is a strong absorption peak near the wavenumber of 1262 $\mathrm{cm}^{-1}$, which is attributed to the absorption of $\mathrm{P}=\mathrm{O}$. Above all, this indicates that MDPA has been successfully grafted onto the Lyocell fiber by HMM. $^{17,18}$

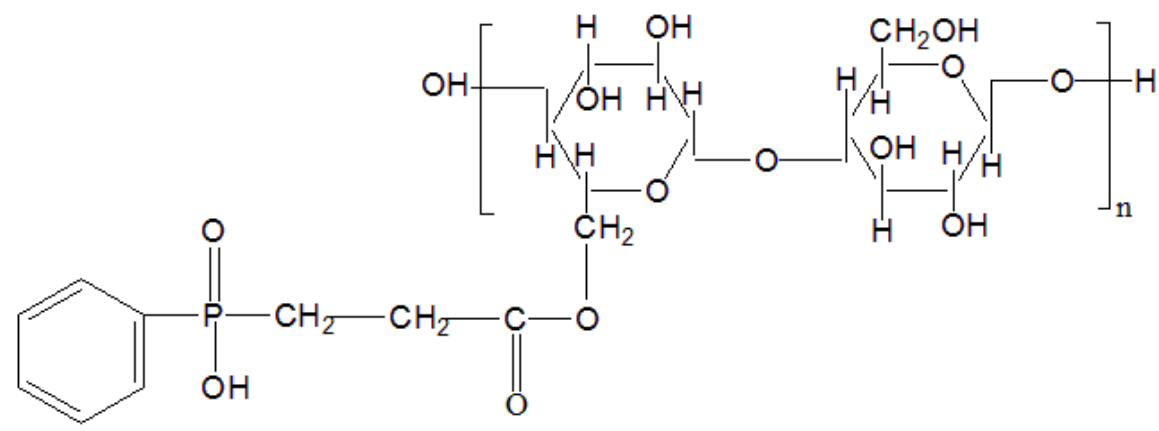

Scheme 1: Structure of flame-retarding Lyocell fibers with CEPPA

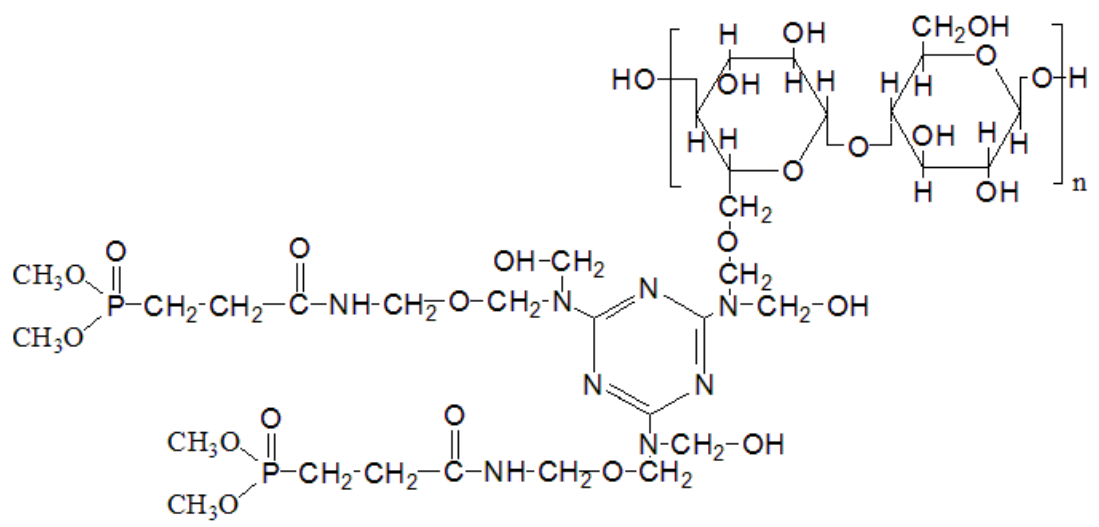

Scheme 2: Structure of flame-retarding Lyocell fibers with MDPA 
Lyocell fibers

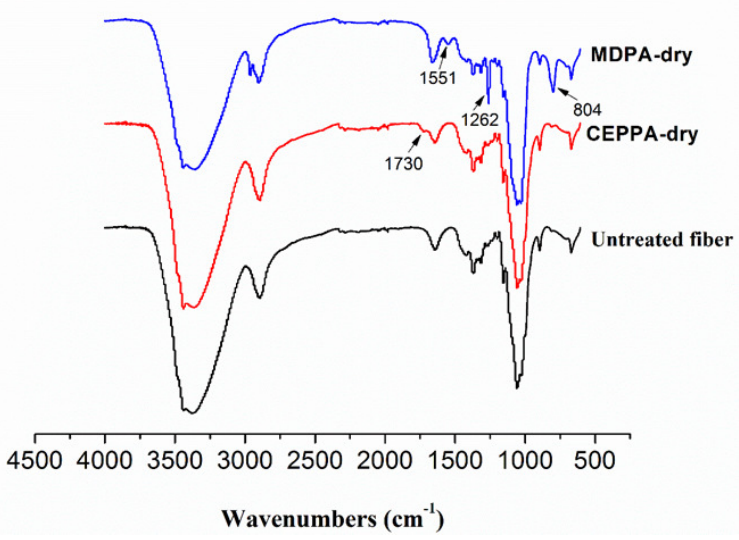

Figure 1: Infrared spectra of Lyocell fiber before and after flame-retardant treatment
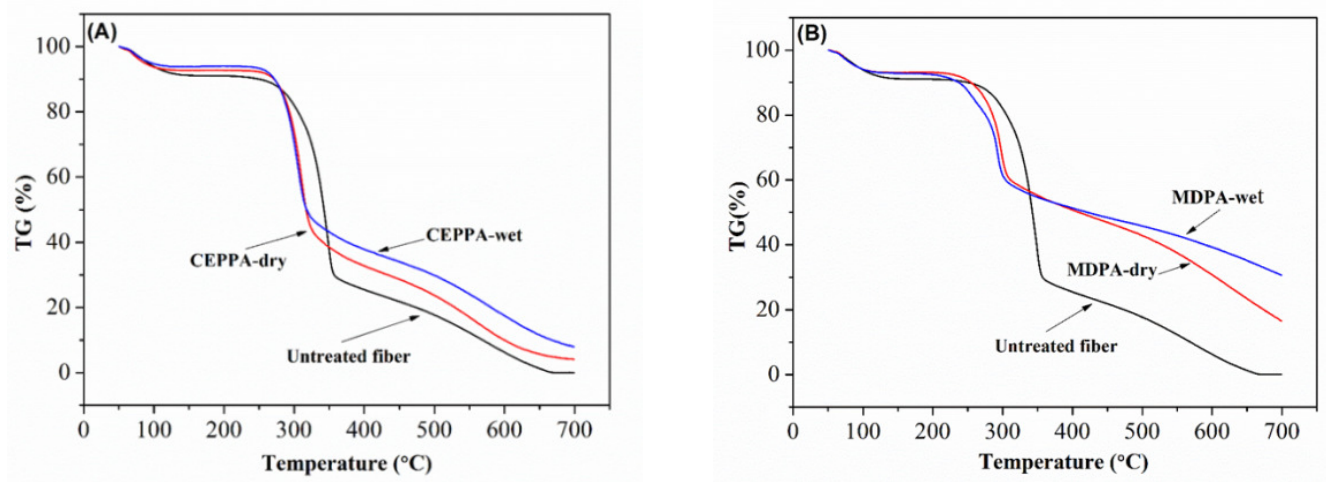

Figure 2: TG curves of Lyocell fiber treated by CEPPA (A) and MDPA (B)

\section{Thermal stability of the fibers}

The thermal stability of the Lyocell fibers treated by CEPPA and MDPA under different conditions was analyzed using a synchronous thermal analyzer. The test results are shown in Figure 2.

It can be seen from Figure 2 that the thermal weight loss rate of the Lyocell fiber treated by CEPPA and MDPA is accelerated, and the thermal decomposition phase of the fiber is advanced. The main reason is that the flame retardants, CEPPA and MDPA, undergo chemical reactions under high temperature conditions, capturing hydrogen and oxygen in cellulose to generate phosphoric acid, metaphosphoric acid, and polymetaphosphoric acid. As a result, the cellulose generates L-glucose and is dehydrated and carbonized, which promotes the thermal degradation and carbonization of the fiber at a lower temperature. The remaining carbon generated further suppresses the generation of flammable gases. In addition, the carbon layer also acts as an oxygen barrier to further reduce the thermal degradation of the internal fibers. It can also be seen from Figure 2 that the residual amount of the fiber without flame-retardant treatment is only $0.06 \%$, and the residual amount of the fiber after the flame-retardant treatment of the dry fiber is $4.10 \%$ (for CEPPA), $16.52 \%$ (for MDPA), respectively. Meahwhile, after the flame-retardant treatment of the never-dried fibers, the residual amounts of the fibers were $7.93 \%$ (for CEPPA) and $30.65 \%$ (for MDPA), respectively, which were $93.41 \%$ (for CEPPA) and $85.53 \%$ (for MDPA) higher than those corresponding to the dry fibers. The above results showed that when two kinds of flame-retardant finishing solutions are used to treat never-dried fibers, the amount of flame retardant grafted to the fibers is higher than that of the dry fibers.

\section{Crystalline properties of the fibers}

The crystal structure of the Lyocell fibers treated with MDPA was analyzed by X-ray diffraction. The test results are shown in Figure 3. 
It can be seen from Figure 3 that the Lyocell fibers treated by MDPA have characteristic diffraction peaks at $2 \theta=12.2^{\circ}, 20.2^{\circ}$, and $21.9^{\circ}$, which correspond to the crystal structure of cellulose II, indicating that the characteristic diffraction peak positions of the treated Lyocell fibers do not change, that is, the main crystal structure of the fiber has not changed, and it is still a cellulose II type crystal structure. ${ }^{19}$ Furthermore, PEAKFIT software was used to perform peak fitting on the spectra in Figure 3 to calculate the crystallinity of the Lyocell fibers treated under different processing conditions, and the results are shown in Table 2. The results show that the crystallinity of the fibers treated by MDPA decreased to varying degrees. In addition, the never-dried Lyocell fiber had a slightly lower crystallinity than the dry fiber after the flameretardant treatment. The main reason is that the never-dried fiber has a more quasi-crystalline structure, therefore, during the flame-retardant treatment, the flame retardant can contact with more cellulose molecules and undergo a graft reaction, which inhibits the movement of cellulose molecular chains, weakens the intermolecular forces, and makes the growth of crystal grains difficult, so the crystallinity of the fibers is reduced.

\section{Mechanical properties of the fibers}

The fiber fineness meter and the monofilament strength tester were used to test the breaking strength and initial modulus of the Lyocell fiber before and after the treatment. The test results are shown in Figure 4.

It can be seen from Figure 4 that, after the flame-retardant treatment, the breaking strength and initial modulus of the Lyocell fibers decreased. The breaking strength of the dry fiber treated with the flame retardants decreased from $3.85 \mathrm{cN} / \mathrm{dtex}$ to $2.44 \mathrm{cN} / \mathrm{dtex}$ (for CEPPA) and $3.24 \mathrm{cN} / \mathrm{dtex}$ (for MDPA), respectively, while the breaking strength of the never-dried fiber after the treatment decreased to $2.24 \mathrm{cN} / \mathrm{dtex}$ (for CEPPA) and $2.79 \mathrm{cN} / \mathrm{dtex}$ (for MDPA), i.e. decreased more than that of treated dry fibers, but still fully meeting the requirements for textiles.

\section{$P$ content and flame retardancy of the fiber}

CEPPA and MDPA flame retardants were used to treat dry and never-dried Lyocell fibers, and the $\mathrm{P}$ content and LOI of the fibers before and after treatment were measured. The results are shown in Table 3.

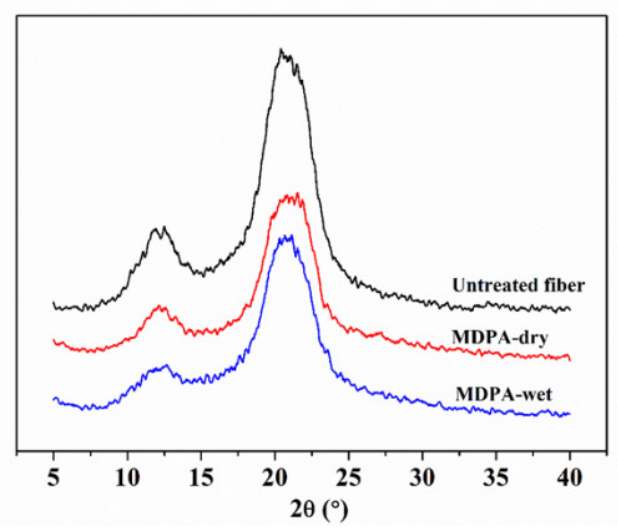

Figure 3: X-ray diffraction patterns of Lyocell fibers treated with MDPA

Table 2

Crystallinity changes of Lyocell fibers treated with MDPA

\begin{tabular}{lc}
\hline Sample code & Crystallinity (\%) \\
\hline Untreated fiber & 58.12 \\
MDPA-dry & 57.83 \\
MDPA-wet & 55.17 \\
\hline
\end{tabular}



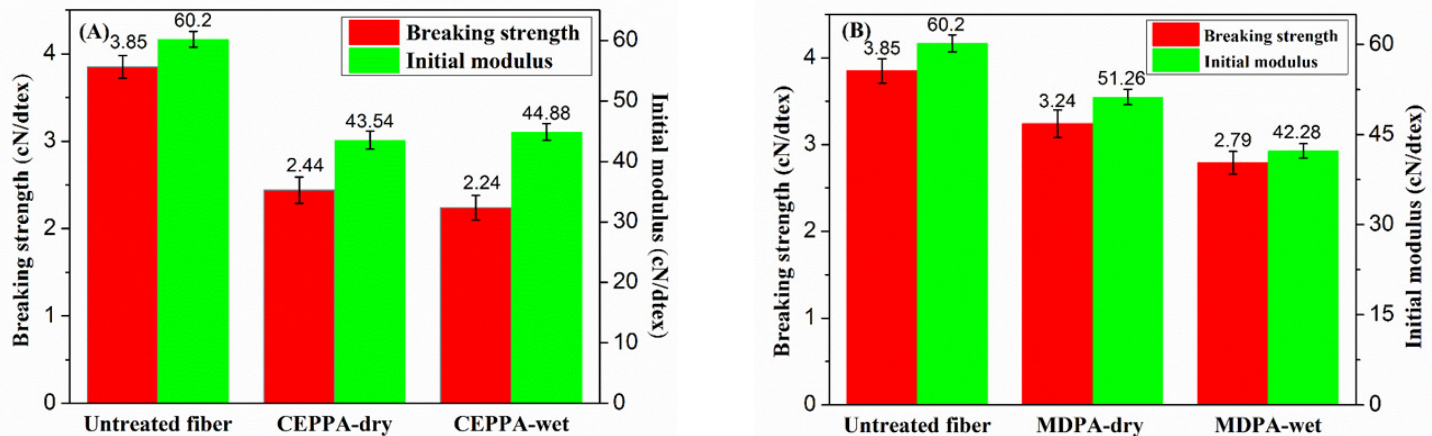

Figure 4: Mechanical properties of Lyocell fiber treated by CEPPA (A) and MDPA (B)

Table 3

$\mathrm{P}$ content and LOI value of Lyocell fiber before and after treatment

\begin{tabular}{lcc}
\hline Sample & Content of P $(\mathrm{mg} / \mathrm{g})$ & LOI $(\%)$ \\
\hline Untreated Lyocell fiber & 0 & 18.9 \\
CEPPA-dry & 5.71 & 24.8 \\
CEPPA-wet & 7.93 & 26.3 \\
MDPA-dry & 12.64 & 34.7 \\
MDPA-wet & 15.24 & 36.1 \\
\hline
\end{tabular}

As can be seen from Table 3, the untreated Lyocell fiber has a LOI of $18.9 \%$ and is extremely flammable. However, the P content and the LOI of the fiber treated by CEPPA and MDPA flame retardants were significantly improved. In particular, the LOI of the Lyocell fiber treated by the flame retardant MDPA reaches more than $34 \%$, which means excellent flame-retardant properties. Upon further comparison of the $\mathrm{P}$ content and LOI of the fibers obtained by treating dry and never-dried fibers with CEPPA and MDPA, respectively, it was found that the $\mathrm{P}$ content and LOI of never-dried Lyocell fiber were higher than those of dry fibers, i.e. the $\mathrm{P}$ content increased by $38.9 \%$ (for CEPPA) and 20.5\% (for MDPA), and the LOI increased by $6.0 \%$ (for CPPA) and $4.0 \%$ (for MDPA), respectively. The main reason should be that the never-dried fiber has a more quasi-crystalline structure, and its aggregation structure is not very tight. During the flame-retardant treatment, the flame-retardant molecules can penetrate more easily into the inner structure of cellulose, and the flame retardant molecules can contact more cellulose molecules and undergo a grafting reaction. Therefore, the experimental results showed that under the same treatment conditions, the flame-retardant effect of the fiber obtained by treating never-dried Lyocell fiber is better, and the energy consumption caused by repeated drying can be reduced. So, other posttreatments of Lyocell fiber, such as dyeing, and immersion of the catalyst for carbon fiber preparation etc., are better to be applied directly on as-spun never-dried fiber, which not only has a good effect, but also can avoid the energy waste caused by repeated drying.

\section{CONCLUSION}

(1) CEPPA and MDPA can react with the hydroxyl group of cellulose and graft onto Lyocell fiber under appropriate conditions, resulting in an increase in the residual amount of the fiber after the thermal degradation and in the flame-retardant performance of the fiber, a slight reduction in crystallinity, and a significant decline in mechanical properties.

(2) Compared with the dry fiber, the P content and LOI of the fiber obtained by treating the asspun never-dried Lyocell fiber with CEPPA and DMPA are significantly higher, but the breaking strength of the fiber decreased more, still fully meeting the requirements for textiles. Therefore, the post-treatment of Lyocell fibers is best to be performed before the fibers are dried.

ACKNOWLEDGMENTS: This work was supported by grants from the National Key R\&D Program of China (2017YFB0309501). 


\section{REFERENCES}

H. B. Öztürk, M. Abu-Rous, B. MacNaughtan, K. C. Schuster, J. R. Mitchell et al., Macromol. Symp., 294, 24

(2010),

https://doi.org/10.1002/masy.200900027

2 P. Weigel, H. P. Fink, E. Walenta and J. Ganster, Cellulose Chem. Technol., 31, 321 (1997), https://www.cellulosechemtechnol.ro/

3 S. A. Mortimer and A. A. Péguy, Cellulose Chem. Technol., 30 117 https://www.cellulosechemtechnol.ro/

4 S. A. Mortimer, A. A. Péguy and R. C. Ball, Cellulose Chem. Technol., 30, 251 (1996), https://www.cellulosechemtechnol.ro/

5 H. Chanzy, M. Paillet and R. Hagege, Polymer, 31, $400 \quad$ (1990), https://doi.org/10.1016/00323861(90)90376-A

6 J. Lenz, J. Schurz and E. Wrentschur, Colloid. Polym. Sci., 271, 460 (1993), https://doi.org/10.1007/Bf00657390

7 H. P. Fink, P. Weigel, H. J. Purz and J. Ganster, Prog. Polym. Sci., 26, 1473 (2001), https://doi.org/10.1016/S0079-6700(01)00025-9

8 M. Y. Wei, G. S. Yang, Y. X. Tian, H. L. Shao et al., Holzforschung, 63, $23 \quad$ (2009), https://doi.org/10.1515/Hf.2009.006

A. Nechwatal, T. Reußmann, C. Hauspurg and M. Nicolai, Melliand China, 6, 10 (2002), https://kns.cnki.net/kcms/detail/detail.aspx?FileName= GFZB200202002\&DbName $=$ CJFQ2002
10 H. H. Zhang, H. L. Shao and X. C. Hu, J. Appl. Polym. Sci., 107, $1738 \quad$ (2006), https://doi.org/10.1002/app.23383

11 C. D. Delhom and L. A. White, Compos. Part B, 41, 475

(2010),

https://doi.org/10.1016/j.compositesb.2009.10.007

12 H. Seddon, M. Hall and A. R. Horrocks, Polym. Degrad. Stabil., 54, $401 \quad$ (1996), https://doi.org/10.1016/S0141-3910(96)00070-5

13 N. Mengal, U. Syed, S. A. Malik, I. A. Sahito et al., Carbohyd. Polym., 153, 78 (2016), https://doi.org/10.1016/j.carbpol.2016.07.074

${ }_{14}$ X. H. Liu, Q. Y. Zhang, B. W. Cheng, Y. L. Ren et al., Cellulose, 25, $799 \quad$ (2018), https://doi.org/10.1007/s10570-017-1550-0

15 G. S. Yang, Y. P. Zhang, M. Y. Wei, H. L. Shao et al., Carbohyd. Polym., 81, $114 \quad$ (2010), https://doi.org/10.1016/j.carbpol.2010.02.003

16 W. D.Wu and C. Q. Yang, Polym. Degrad. Stabil., 91, 2541 (2006), https://doi.org/10.1016/j.polymdegradstab.2006.05.010

17 J. T. Hu, Y. N. Yao, X. S. Liu, Y. H. Ao and H. X. Zhang, Fire Mater., 33, $145 \quad$ (2009), https://doi.org/10.1002/fam.990

${ }_{18}$ Z. Y. Yang, X. W. Wang, D. P. Lei, B. Fei and J. H. Xin, Polym. Degrad. Stab., 97, 2467 (2012), https://doi.org/10.1016/j.polymdegradstab.2012.05.023 19 R. G. Liu, X. C. Hu and T. L. Zhang, J. China Text. Univ., $\quad$ 24, 7 (1998), https://kns.cnki.net/kcms/detail/detail.aspx?FileName= DHDZ199804001\&DbName=CJFQ1998 